\title{
On Comparative Analogy of Academic Performance Quality Regarding Noisy Learning Environment versus Non-properly Prepared Teachers Using Neural Networks' Modeling
}

\author{
Hassan M. Mustafa and Ayoub Al-Hamadi
}

\begin{abstract}
This piece of research presents analytical evaluation of comparative analogy between two educational phenomena considering academic performance (achievement) observed inside our classrooms. These two phenomena are: the effect of noisy learning environment on educational field academic achievement quality. In addition to the impact of interactive teaching by non-properly prepared teachers on academic performance.

The comparative analogy investigated herein, is presented systematically via adopting Artificial Neural Networks' (ANNs) modeling. By more details: noisy data considered as the main cause of environmental annoyance which negatively affects the quality of academic performance. Furthermore, considering student's learning ability problem faced by non-properly prepared teachers during their academic tutoring performance inside classrooms,.

On the basis of the adopted supervised learning ANN model, two basic neural networks' parameters have been explicitly considered. That's while running of presented simulation program, both parameters are: learning rate value $(\eta)$ and gain factor value $(\lambda)$.
\end{abstract}

Index Terms-Artificial neural networks models, academic performance, signal to noise ratio.

\section{INTRODUCTION}

The field of the learning sciences is represented by a growing community internationally. Many educational experts now recognize that conventional ways of conceiving learning are facing increasing challenges in this time of rapid Technological, and social changes. Recently, that increasing challenges have adopted a novel trend of neural networks' application in educational systems. Which supported by what had been announced last decade (1990-2000) (in USA) that called as Decade of the brain [1], [2].

In the two contexts of recent rapid Technological / social changes, and educational application of Artificial Neural Networks $\left(\mathrm{ANN}^{\mathrm{s}}\right)$. Realistic modeling trend regarding main human brain functions (learning and memory) has been considered. Additionally, analysis and evaluation of educational phenomenal issues have been adopted by most of educationalists as well as neural networks' experts [3]-[5].

Manuscript received May 20, 2015; revised August 18, 2015.

Hassan M. Mustafa is with Computer Eng. Department, Faculty of Engineering. Al-Baha University (K.S.A). He is on leave from Educational Technology Department Faculty of Specific Education-Bana University Egypt (e-mail: prof.dr.hassanmoustafa@gmail.com).

Ayoub Al-Hamadi is with Institute for Electronics, Signal Processing and Communications (IESK) Otto-von-Guericke-University Magdeburg Germany (e-mail: Ayoub.Al-Hamadi@ ovgu.de).
Interestingly, that approach of recent trend at academic field results in a big critical educational question, which splitted into two interrelated questions: how realistic simulation using ANN modeling capable to evaluate learning process convergence considering noisy environment? [5], [6] \& how this process may be quantitatively affected by contaminated noisy information submitted by a non-properly prepared teacher to students in classrooms? [7]. This paper is organized by five sections as follows. An introduction of presented work is given at the first section. At the next second section revising of the interactive educational process is introduced via its three subsections $(A \& B)$. A general conceptual modeling of interactive learning process is presented at the first subsection $(A)$. At the second subsection $(B)$, some details for mathematical formulation of interactive learning process are introduced. The impact of noisy data on students' academic performance is introduced in more details at the third section which composed of three subsections $(A \& B \& C)$. At subsection $(A)$, the simulation results presenting the Effect of Noisy Learning Environment on rate of academic (learning) performance is given. Derivation of the relation between learning rate parameter $\eta$ to Noise Power $\sigma$ is presented at subsection $(B)$. This subsection illustrates that contaminated recognition process by intended noisy power levels (signal to noise ratios) negatively effects academic performance. It has reflected mapping analogy between contaminated recognition processes versus non-properly prepared teacher's influence of on academic learning performance in classrooms. At subsection (C), More Details about Noisy Learning Effect on Academic Performance (Neurophysiological View) are presented. A brief fourth section considers the analogy between the negative effects of non-properly prepared teachers on academic performance versus the influence of $(\mathrm{S} / \mathrm{N}) \mathrm{ratio}$.

The obtained simulation results after running the adopted $\left(\mathrm{ANN}^{\mathrm{s}}\right.$ ) model are introduced along with a comprehensive analysis at the fifth section. Finally, some interesting conclusive remarks are given at the last sixth section.

\section{Revising OF EduCATIONAL Process Modeling}

This revising section introduces the conceptual basis of teaching / learning process and illustrates its realistic interactive modeling via four subsections as follows.

At the subsection $A$, an overview generalized brief of the block diagram describing interactive teaching / learning process is given. More analysis about the interactive learning process is presented at subsection $B$. In $A$ detailed 
mathematical formulation considering either bidirectional communication between a teacher and his learners (supervised) or self-organized (unsupervised) Kohonen learning by interaction with environment is introduced at subsection $C$.

\section{A. Modeling of Interactive Learning Process}

Referring to Fig. 1, it illustrates a general view of a teaching model qualified to perform simulation of above mentioned brain functions. Inputs to the neural network teaching model are provided by environmental stimuli (unsupervised learning). However, correction signal(s) in the case of learning with a teacher given by output response(s) of the model that evaluated by either the environmental conditions (unsupervised learning) or by supervision of a teacher. Furthermore, the teacher plays a role in improving the input data (stimulating learning pattern) by reducing the noise and redundancy of model pattern input. That is in accordance with tutor's experience while performing either conventional (classical) learning or Computer instruction learning. Consequently, he provides the model with non-redundant cleared data maximizing its signal to noise ratio in according to tutor's experience [8]. Conversely, in the case of unsupervised/self-organized learning, this is based upon either Hebbian rule [9], or supervised interaction with environment [10]. Interestingly, from the interactive learning P.O.V., cognitive brain function at [11]-[14], is implicitly formulated mathematically by two equations (7) and (8) presented at the next section (B).

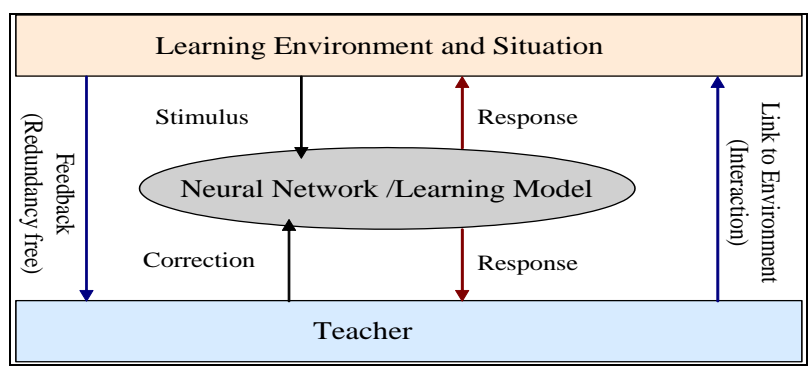

Fig. 1. Illustrates generalized simple block diagram for interactive learning process.

\section{B. Mathematical Formulation of Interactive Learning}

The presented model given in Fig. 2 simulates simply two diverse learning paradigms. It presents realistically both paradigms: by interactive learning / teaching process, as well as other self-organized (autonomous) learning. By some details, firstly is concerned with classical supervised by a tutor observed in our classrooms (face to face tutoring). Accordingly, this paradigm proceeds interactively via bidirectional communication process between a teacher and his learners (supervised learning) [15].

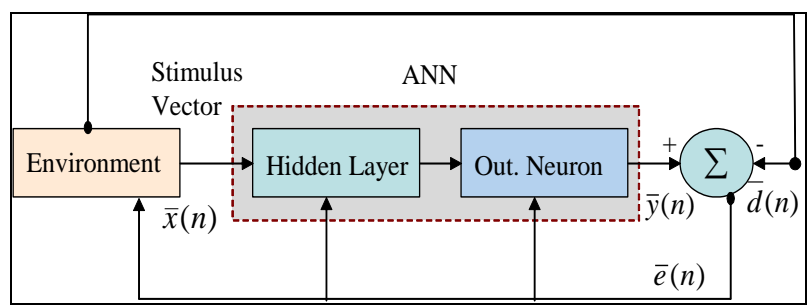

Fig. 2. Generalized ANN block diagram simulating two diverse learning paradigms adapted from [15].
Referring to above Fig. 2; the error vector $\bar{e}(n)$ at any time instant $(n)$ observed during learning processes is given by:

$$
\bar{e}(n)=\bar{y}(n)-\bar{d}(n)
$$

where $\bar{e}(n) \ldots \ldots$ is the error correcting signal that adaptively controls the learning process,

$\bar{y}(n) \ldots \ldots$ is the output obtained signal from ANN model, and $\bar{d}(n) \ldots \ldots$ is the desired numeric value(s).

Moreover, the following four equations are deduced to illustrate generalized interactive learning process. These equations are commonly well valid for either guided with a teacher (supervised) or self-learning without a teacher (unsupervised):

Equation (2) considers the scalar product of two vectors the input vector $(X)$ and internal weight vector $(W)$ computed at the time instant $(n)$. It is noticed that both are associated to neuron $(k)$, and each has the same dimension (number of vector's components). The output of this neuron is given by equation (3). Which originated from the hyperbolic tangent function deduced from classical sigmoid function.

Equation (4) computes the error value which controls the guided learning process (supervised with a teacher) and so it does not valid in case of unsupervised (learning without a teacher).

The dynamic learning law at two subsequent time instances (n) \& $(n+1)$ is shown by equation (5).

$$
\begin{gathered}
V_{k}(n)=X_{j}(n) W_{k j}^{T}(n) \\
Y_{k}(n)=\phi\left(V_{k}(n)\right)=\left(1-\mathrm{e}^{-\lambda V_{k}(n)}\right) /\left(1+\mathrm{e}^{-\lambda V_{k}(n)}\right) \\
e_{k}(n)=\left|d_{k}(n)-y_{k}(n)\right| \\
W_{k j}(n+1)=W_{k j}(n)+\Delta W_{k j}(n)
\end{gathered}
$$

where $X$ is input vector and $W$ is the weight vector. $\varphi$ is the activation function. $Y$ is the output. $e_{k}$ is the error value and $d_{k}$ is the desired output. Note that $\Delta W_{k j}(n)$ is the dynamical change of weight vector value. Above four equations are commonly applied for both learning paradigms: supervised (interactive learning with a tutor), and unsupervised (learning though student's self-study). The dynamical changes of weight vector value specifically for supervised phase is given by:

$$
\Delta W_{k j}(n)=\eta e_{k}(n) X_{j}(n)
$$

where $\eta$ is the learning rate value during the learning process for both learning paradigms. At this case of supervised learning, instructor shapes child's behavior by positive / negative reinforcement Also, Teacher presents the information and then students demonstrate that they understand the material. At the end of this learning paradigm, assessment of students' achievement is obtained primarily 
through testing results. However, for unsupervised paradigm, dynamical change of weight vector value is given by:

$$
\Delta W_{k j}(n)=\eta Y_{k}(n) X_{j}(n)
$$

Noting that $e_{k}(n)$ equation (6) is substituted by $y_{\mathrm{k}}(n)$ at any arbitrary time instant $(n)$ during the learning process. Instructor designs the learning environment.

\section{REVISING OF NOISY LEARNING ENVIRONMENT [2]}

Noisy data at educational field classrooms observed to be the main phenomenal cause that results in negatively influences on quality of academic performance. In other words, noisy learning environment results in worst learning/teaching process and less students' focusing on lessons in classrooms. At the following subsection A, some interesting simulation results are presented.

\section{A. Effect of Noisy Learning Environment}

At educational field practice, it has been found that children learn in a variety of ways such as teacher-centered learning and teaching style [16] Traditional teaching is concerned with the teacher being the controller of the learning environment. A major factor in determining the nature of the physical classroom environment is the type of learning that the teacher wishes to encourage. This is directly related to teaching style. [17]. These different ways in which a child learns is partially related to the type of learning environment available [18]. These learning environments may also affect the child's ability and motivation to learn.

In nature, ideal (noiseless) learning environment is not available in practice. Usually, it is environmental learning data is vulnerable to contaminations by either external or internal noisy conditions. So, learning/teaching processes in our classrooms, for either suggested topics, have to be accomplished under influenced effect of some environmental noise [19]. Therefore considerable attention has been paid in this subsection to report the effect of either noisy CAL environment or noisy teacher on learning convergence time. The simulation results were obtained via association between the two input stimuli (visual and auditory) following classical conditioning learning [16], [17]. Obtained results for Optical Character Recognition (OCR) under different noise levels are given in a tabulated form as in Table I.

Practically, the best way to teach children how to read is carried out under the effect of less noisy data. The noise effect is measured by signal to noise ratio value $(\mathrm{s} / \mathrm{n})$ versus the number of training cycles (t). Conclusively, an interesting remark observed considering relation betweennumber of training cycles values (convergence learning time), and noisy environmental data in case of application of adopted ANN learning model. The convergence time cycles $(t)$, of learning process is inversely proportional to signal to noise ratio values, $(\mathrm{s} / \mathrm{n})$ and learning rate values. On the other hand, it is directly proportional to noise power value(s) [18]. Additionally, the evaluated relation between learning rate values and noisy data (tutor) appeared considering unsupervised learning. The convergence time of learning process is reached after 47, 62, and 85 training cycles when noise power is $0.05,0.1$ and 0.2 , respectively, as shown at Table I. Running the ANN simulation program with three numerical learning rate values $(0.2,0.1$ and 0.05$)$ resulted in the training cycles $(3,5$, and 10$)$ respectively. Fig. 7 shows the statistical distribution for the relation between number of training cycles and learning rates. This distribution is seems to be similar to Gaussian (normal) as it has a bell shape, which proved the realistic of the developed model.

Referring to Fig. 4, the threenoise power values $\sigma(0.2,0.1$, and 0.05$)$ are correspondingto the three noisy values observed respectively as $\mathrm{S} / \mathrm{N}$ ratios $(5,10$, and 20). Which contaminatedenvironmentallearning information/data applied in classrooms. Interestingly, it is noticed that by the increase of $\mathrm{S} / \mathrm{N}$ ratio values (more properly prepared teacher) results in improvement of learning rate parameter values $\eta$.

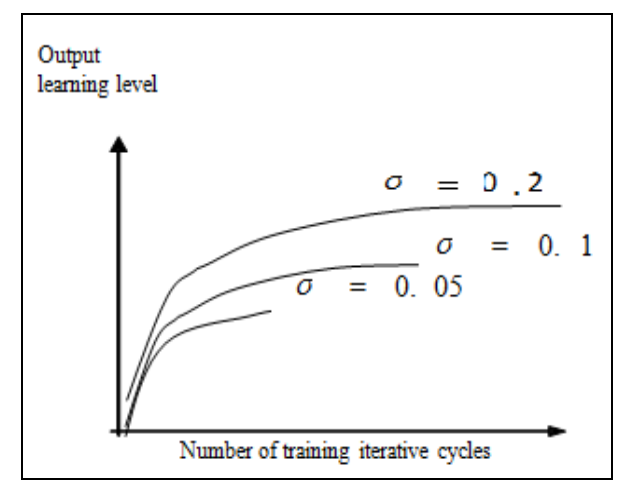

Fig. 3. Relation between noise power $(\sigma)$ that represents non-properly prepared (noisy) learning process convergence, adapted from [19].

\section{B. Relationship between Noise Power $\sigma$ versus Learning Rate Parameter Value $\eta$}

Referring to Fig. 4, the three various values of noisy power $\sigma(0.2,0.1$, and 0.05$)$ are respectively corresponding to contaminated noisy leaning environmental information/data which having $\mathrm{S} / \mathrm{N}(5,10$, and 20). Moreover, by increasing of $\mathrm{S} / \mathrm{N}$ ratio, more improvement of learning rate parameter value $\eta$ is observed. In other words, that convergence learning time measured by number of interactive cycles is inversely proportional to the learning rate values.

As an illustrative numerical example, simulation results are given at Fig. 5 in below. It clarifies the statistical distribution for measured values students' academic achievement considering learning convergence (response time) associated to samples students' group. That is considering two different learning rate values $(\eta=0.1 \& \eta=0.5)$. As more illustrative view concerned with relation between learning rate value parameter $\eta$, and learning convergence time is presented by simulation results of two learning rate values versus response time parameter given at Fig. 6. These results obtained after considering supervised (error correction) learning paradigm (following equation (6) given in the above.

Furthermore, in Fig. 7 simulation results are presented via statistical distribution for students' achievements (normalized outcomes' marks) versus the frequency of occurrence (for various normalized achievement outcomes' values) at two different learning rate values $(\eta=0.1$ and $\eta=0.5)$. The resulting statistical values' distribution observed to have a bell shape form which approximately similar tonormal 
(Gaussian) distribution. Interestingly, that shape is also introduced at Fig. 6.

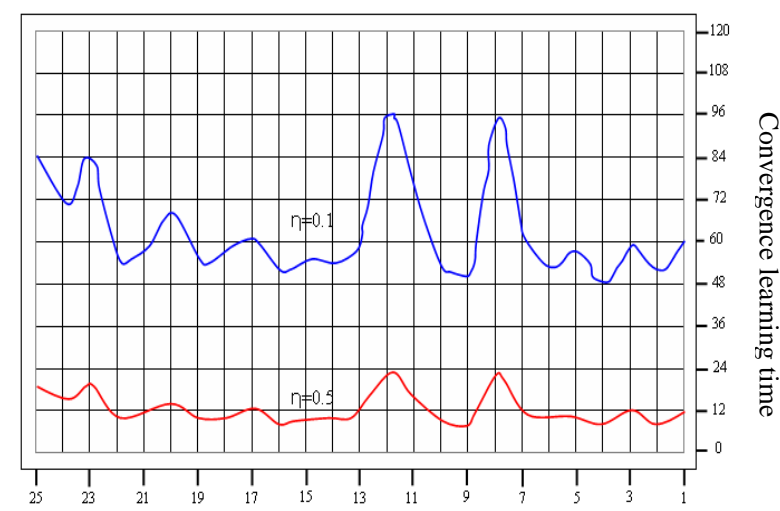

Samples of students group

Fig. 5. Illustrates the effect of two different values of learning rate parameters $\eta$ values on the convergence learning time. $\eta$ values 0.1 (Upper curve) \& 0.5 (Lower curve) noting that time response obeys Gaussian distribution in an approximate manner.

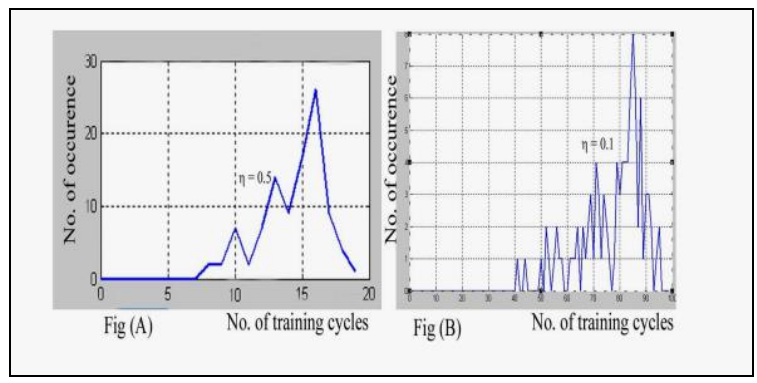

Fig. 6. Illustrates the statistical distribution in relation with two learning rate values of parameter and learning response(convergence) time at Fig (A) \& Fig (B) corresponding to two different learning rate values $\eta 0.5 \& 0.1$ respectively.

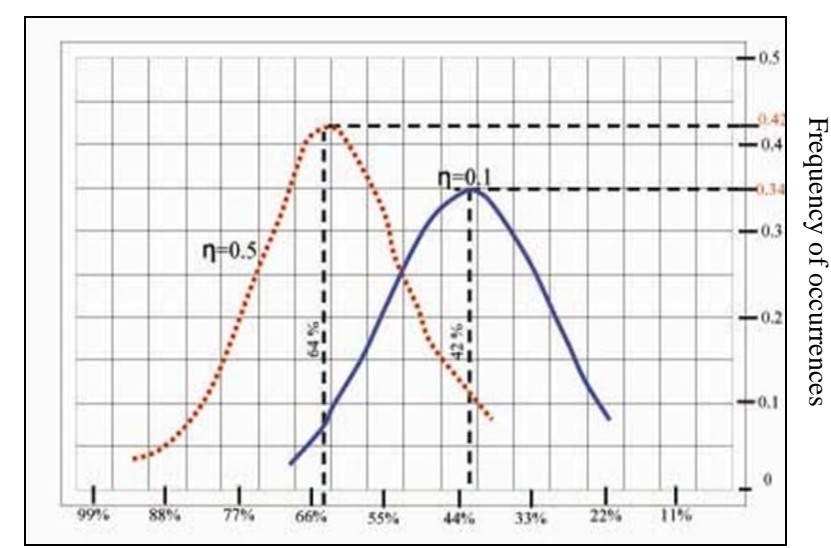

Fig. 7. Illustrates Simulation results presented by statistical distribution for students' achievements versus the frequency of occurrence for various normalized achievements values, at two different learning rate values $(\eta=$ 0.1 and $\eta=0.5)$. Adapted from [20].

\section{More Details about Noisy Learning Effect on Academic Performance (Neurophysiological View)}

In educational field practice-from neurophysiological P.O.V., performing of interactive learning processes utilizes two basic and essential brain cognitive functions. Both functions are originated from performing efficiently interactive processes learning / teaching in accordance with behaviorism approach [13], [14] as follows. Firstly, pattern classification /recognition function based on visual /audible interactive signals stimulated in classrooms by teachers. Secondly, associative memory function is used which originally based on Pavlovian classical conditioning motivated by Hebbian learning rule (details are given at the third section). Referring to Fig. 2, it illustrates teaching model that relevantly qualified in performing brain function stimulation. In case of learning with a teacher, the correction signal (difference between observed learning outcome and the desired output) is provided by responsive output action of the suggested model. Interestingly, teacher plays a role in improving the input data (stimulating learning pattern), by reducing noise and model's Redundancy of pattern input. Regarding teacher's experience concerned with conventional (classical) learning methodology; after analysis of obtained realistic simulation results, two practical issues were observed. Firstly, the model given at Fig. 1 can be provided with cleared data by maximizing its signal to noise $\mathrm{S} / \mathrm{N}$ ratio. Such better $\mathrm{S} / \mathrm{N}$ ratio in noisy learning environment results in improvement of learning performance quality. In other words, better $\mathrm{S} / \mathrm{N}$ ratio results in less number of training cycles, desired learning convergence could be attained (as shown at Table I). Similarly, improvement of learning rate values results in better learning performance indicated by decreased learning response (convergence) time. That improvement illustrated well by simulation results presented graphically at Fig. 2 and tabulated (on the average) at Table II. Secondly, tutors' experience observed to be transferred via a link to children's brain model (ANN) as a correcting stimulating signal. So, that experience may be capable of increasing number of neurons contributing to learning process convergence. Specifically, those increased number of neurons are placed (from neurobiological brain structure) at hippocampus brain area.

TABLE I: THE EFFECT OF SIGNAL TO NOISE RATIO ON LEARNING CONVERGENCE TIME AND NON-PROPERLY PREPARED TEACHERS, ADAPTED FROM [19]

\begin{tabular}{|l|c|c|c|}
\hline \multicolumn{1}{|c|}{ Signal to Noise Power Ratio of Input Data } & 5 & 10 & 20 \\
\hline Noise Power $\boldsymbol{\sigma}$ in Learning Environment & 0.2 & 0.1 & 0.05 \\
\hline Convergence Learning Time (cycles) & 85 & 62 & 47 \\
\hline
\end{tabular}

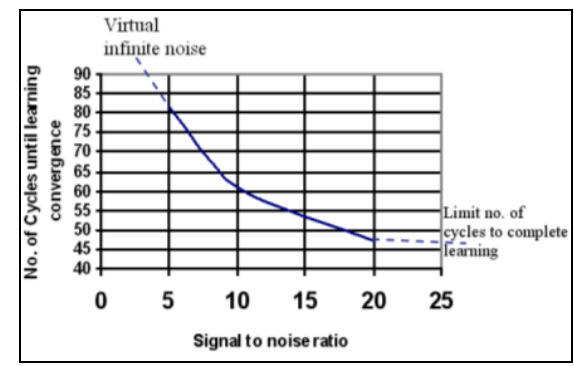

Fig. 8. Graphical representation for observed learning performance referred to above Table I.

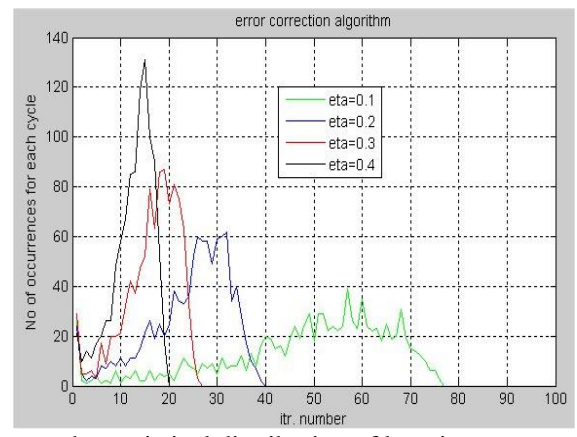

Fig. 9. Illustrates the statistical distribution of learning convergence time for different learning rate values (eta) adapted from [6]. 
TABLE II: THE RELATION BETWEEN LEARNING RATE VALUES AND CONVERGENCE LEARNING TIME

\begin{tabular}{|c|c|c|c|c|}
\hline $\begin{array}{l}\text { learning rate values } \\
\eta\end{array}$ & 0.1 & 0.2 & 0.3 & 0.4 \\
\hline $\begin{array}{c}\text { Average Learning } \\
\text { Convergence Time } \\
\text { (cycles) }\end{array}$ & 55 & 27 & 17 & 13 \\
\hline
\end{tabular}

\section{NON-PROPERLY PREPARED TEACHER VERSUS NOISY LEARNING ENVIRONMENT}

Non-properly prepared Teacher's performance is analogous to signal to the influence of $(\mathrm{S} / \mathrm{N})$ ratio on academic performance. That analogy is illustrated well by referring to generalized simple block diagram for interactive learning process presented at Fig. 1. Therein, teacher's correction signal should have been erroneous in accordance with level of non-proper preparation. In other words, that non-proper preparation level quantitatively measured according to signal to noise $(\mathrm{S} / \mathrm{N})$ ratio or equivalently the additive noise power $(\sigma)$ to the sensory clear (ideal) signal. Consequently, the time response measured by number of training cycles $(n)$ \{defined at the second (B) section at equation (6) $\}$ should have been increased until reaching learning convergence at the instant $(n)$ when

$$
\Delta W_{k j}(n)=0
$$

The given condition by equation (8) could be fulfilled only if the desired output learning has been obtained after some number of training cycles (response time). Therefore, the impact of interactive non-properly prepared (noisy) teacher on learning convergence time is given at Table I. It is adapted from simulation findings published at [19]. Conclusively, it is observed during interactive learning process that: teaching/learning environment with decreasing $\mathrm{S} / \mathrm{N}$ ratio results in decreasing of learning rate parameter value $\eta$. At the next subsection the interrelation between noise power value $(\sigma)$ and learning rate parameter $(\eta)$ is presented. Fig. 3 Graphical presentation of learning performance considering non-properly prepared (noisy) teacher by referring to above Table I.

\section{A COMPREHENSIVE OVERVIEW ON SIMULATION RESUlTS}

Referring to [5]-[7], after running of realistic simulation program that adopts the competitive learning law of Kohonen's self-organized learning [15], [21]. These three changedNoise power values $\sigma(0.2,0.1$, and 0.05$)$ are respectively corresponding to noisy environmental values of $\mathrm{S} / \mathrm{N}$ (5, 10, and 20). Moreover, they observed to be in correspondence with the three learning rate values $\eta(0.05$, 0.1 , and 0.3 ) respectively. Furthermore, after running of the suggested realistic simulation program, it results in the set of three distribution curves depicted at Fig. 11.

Referring to Fig. 12, It is worthy to note that statistical variations (on the average) relating learning rate values versus corresponding selectivity convergence (response) time. That time is measured by the number of iteration cycles. Obtained output results (of response time) corresponding to the learning rate values $(0.1,0.2,0.4,0.6$, and 0.8$)$, are given respectively, as $(330,170,120,80$, and 40$)$ iteration training cycles. Conclusively, convergence time (number of training cycles) is inversely proportional to the corresponding learning rate values. Moreover, it is an interesting remark that under more noisy environmental conditions, learning rate tends to have lower value. Conversely, creatures performing learning rate improvement by interaction with environment imply increase of their stored experience. Consequently, such creatures have become capable of responding spontaneously to input environmental stimuli in optimal manner [10].

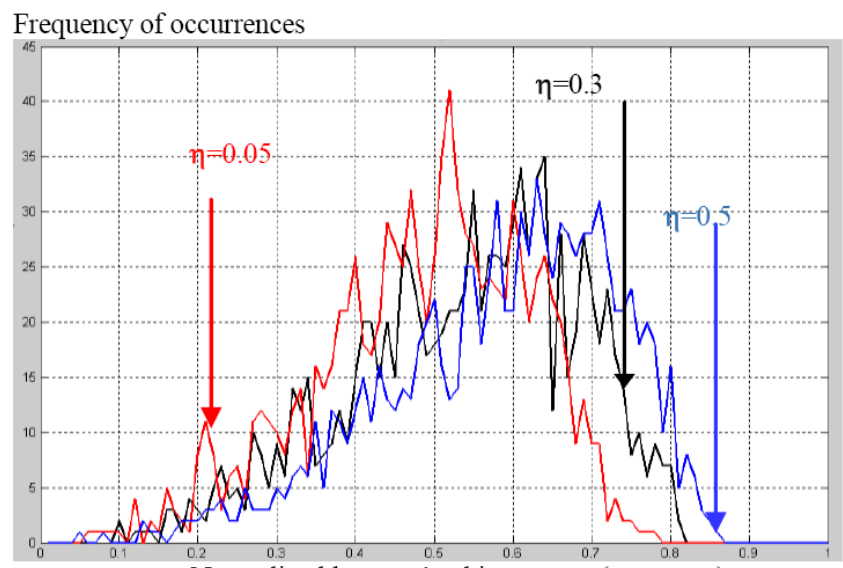

Normalized learners' achievements (outcomes)

Fig. 11. The three changes of noise power values $\sigma(0.2,0.1$, and 0.05$)$ in noisy environment considered to be in correspondence with three learning rate values $\eta(0.05,0.3$, and 0.5$)$

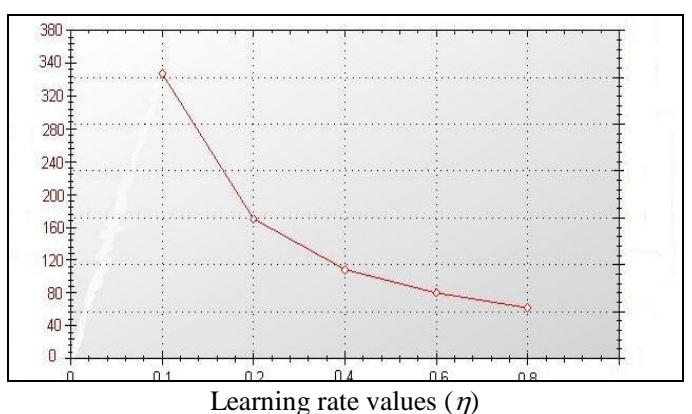

Fig. 12. Illustrates the average of statistical distribution for learning response time (number of iteration cycles) for different learning rate values $\eta$.

\section{CONCLUSIONS, Discussions, AND FUTURE WORK}

Noisy data considered as main cause of environmental annoyance and it negatively affects the quality of life of a large proportion of the population. Herein, this work illustrates the analogy between noisy data learning in Artificial Neural Networks models versus the effect of physical environment on quality of education in classrooms. Interestingly, it is observed thatimpact of interactive non-properly prepared to have negative effect on educational process similar to the noisy environmental learning effect. Those obtained interesting results have been considered as a guiding to challenging research works research approach for more elaborate investigation of observed educational phenomena issues [2].

Generally, at any level of education, school bears their responsibility in order to create relevant enhancing of learning environment. Hat is based on modern skills and knowledge and facilitates students' understanding of the world of 
technology. That learning environment is regarded as the ensemble of the intellectual, social, and physical environments. Accordingly, schools' responsibility have to take into account students' developed learn performance aside from noisy contaminated (undesirable) effects on created learning environment. This work illustrates clearly the analogous undesirable effect observed by effects of both overcrowded classroom as well as Cocktail Party Problem on learning performance phenomenon. The learning environment supports the student's development into an independent and active learner, carries the basic values of basic education and the school's mental attitude, and preserves and refines the traditions of the region and the school community. Furthermore, the learning environment creates prerequisites and conditions for acquiring a subject as well as for the development of the student's personality. Interestingly, in ANN context, the two parameters: Learning rate and Gain factor are considered by the presented simulated comparative study. Accordingly, interesting simulation results have been obtained by the end conclusion of this work declaring the interrelation between learning rate values versus different noisy levels. As well as, the effect of intrinsic individual children's differences (gain factor values) on selective attention performance is presented. Furthermore, the work illustrates specifically the analogous effect between Artificial Neural Networks modelling of noisy audible data (education in classrooms), versus the noisy physical visual data such as Optical Character Recognition (OCR).The overcrowding in classrooms shown to have negative effect on educational process similar to the noisy learning environmental effect. The interesting results have been obtained indicating an extendable future challenging research. In future, this work is recommended to be extended by more elaborate practical educational field application, in order to investigate systematically both observed educational phenomena presented herein.

\section{REFERENCES}

[1] White House OSTP Issues Decade of the Brain Report, Maximizing Human Potential: 1990-2000. [Online]. Available: http://www.neurology.org/content/40/2/321.extract

[2] K. Morris, "Advances in 'brain decade' bring new challenges," LACENT Journal, vol. 355, no. 9197, p. 45, January 1, 2000.

[3] B. S. Arons. (June 18, 1997). Parity in coverage of mental health services in an era of managed care. Summary of Presentations. [Online]. Available: http://www.loc.gov/loc/brain/summary2.html

[4] H. M. H. Mustafa, "On quantified evaluation of noisy data impact on children's mental development using artificial neural networks," the presented at 6th International Conference of Education, Research and Innovation held in Seville (SPAIN), November 18-20, 2013.

[5] H. M. H. Mustafa, "Comparative analogy of overcrowded effects in classrooms versus solving 'cocktail party problem' (neural networks approach)," presented at 8th International Technology, Education and Development Conference, March 11-12, 2014.

[6] H. M. Mustafa, A. Al-Hamadi, and M. H. Kortam, "On assessment of teaching a mathematical topic using neural networks models (with a case study)," presented at Conference of Technology and Its Integration into Mathematics Education Time 2010, Malaga, Spain, July 6-10, 2010.

[7] H. M. H. Mustafa, "On analysis and evaluation of non-properly prepared teachers' implication on students considering optical character recognition (OCR) (neural networks' modeling approach)," The International Journal of Computer Science and Electronics Engineering (IJCSEE), vol. 3, issue 2, ISSN 2320-4028, 2015.

[8] Signal to noise ratio definition. Margaret Rouse. [Online]. Available: http://searchnetworking.techtarget.com/definition/signal-to-noise-rati o
[9] D. O. Hebb, "The organization of behavior," A Neuropsychological Theory, New York, Wiley, 1949.

[10] M. Fukaya, "Two level neural networks: Learning by interaction with environment," San Diego, 1988.

[11] H. M. Hassan, "On comparison between swarm intelligence optimization and behavioral learning concepts using artificial neural networks (an over view)," presented at the 12th World Multi-Conference on Systemics, Cybernetics and Informatics: WMSCI 2008. The 14th International Conference on Information Systems Analysis and Synthesis, Orlando, Florida, USA, 2008.

[12] H. M. Hassan, "On evaluation of virtual improvement of learning creativity by application of computer assisted learning using artificial neural networks modeling," presented at Sixth Annual International Conference on Remote Engineering and Virtual Instrumentation REV2009, University of Bridgeport, CT, USA, June 18-22, 2009.

[13] H. M. Hassan, "On analysis of quantifying learning creativity phenomenon considering brain synaptic plasticity," presented at WSSEC08 Conference, Derry, Northern Ireland, August 18-22, 2008.

[14] H. M. Hassan, "On simulation of adaptive learner control considering students' cognitive styles using artificial neural networks (ANNs)," presented at CIMCA, Austria, Nov. 28-30, 2005.

[15] S. Haykin, Neural Networks, Englewood Cliffs, NJ: Prentice-Hall, 1999.

[16] Tradtea. http://ehlt.flinders.edu.au/education/DLiT/2002/environs/scott/tradtea c.htm

[17] Learnty [Online]. Available: http://ehlt.flinders.edu.au/education/DLiT/2002/environs/scott/learnty p.htm

[18] Lenvhome. [Online]. Available: http://ehlt.flinders.edu.au/education/DLiT/2002/environs/suyin/Lenvh ome.htm

[19] M. A. Ghoaimy, A. M. Al-Bassiouni, and H. M. Mustafa, "A learning of neural networks using noisy data," presented at Second International Conference on Artificial Intelligence Application, Cairo, Egypt, pp. 389-399, 1994.

[20] H. M. Hassan and A. Al-Hamadi, "On teaching quality improvement of a mathematical topic using artificial neural networks modeling" (with a case study)," presented at the10th (Anniversary!) International Conference Models for Developing Mathematics Education, Dresden, Saxony, Germany, September 11-13, 2009.

[21] T. Kohonen, Self-organization and Associative Memory, New York, Springer, 1984.

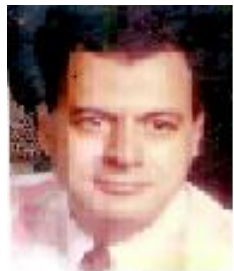

Hassan M. H. Mustafa was born in Cairo, on October 1, 1947. He received his B.Sc. degree and M.Sc. degree in electrical engineering from Military Technical College Cairo-Egypt in 1970, and 1983 respectively. He received his $\mathrm{Ph} . \mathrm{D}$. degree at computer engineering and systems in 1996 from Ain Shams University, Faculty of Engineering Cairo, Egypt. Currently, he is an associate professor with Computer Engineering Department, Al-Baha University K.S.A. He is a member with a set of scientific, engineering, and educational technology societies, such as IIIS (International Institute of Informatics and Systemics) etc. $\mathrm{He}$ is a senior member at International Economics Development Research Center (IEDRC) organization. Furthermore, he has been appointed as a member of technical comity for artificial neural networks research work at IASTED organization during the period (2009-2012). His interest fields of research are artificial neural networks, natural inspired computations, and their applications for simulation, modeling and evaluation of learning processes / phenomena. He is an author / coauthor for more than 90 published publication papers \& technical reports \& books.

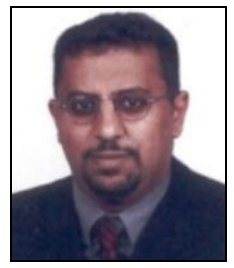

Ayoub Al-Hamadi was born in Yemen. He received the master's degree in electrical engineering and information technology in 1997, the Ph.D. degree in technical computer science in 2001, the habilitation degree in artificial intelligence in 2010, and the Venia Legendi degree in pattern recognition and image processing from Otto von Guericke University Magdeburg, Magdeburg, Germany.

He became a professor of Neuro-Information Technology with Otto von Guericke University Magdeburg in 2008. He has been a junior research group leader with the Institute for Electronics, Signal Processing and Communications, Otto von Guericke University Magdeburg, since 2002. He has authored over 280 articles in peer-reviewed international journals, conferences, and books. 